\title{
Crecimiento guiado por exportaciones y metas de inflación en México: una apuesta en contra del crecimiento ${ }^{1}$
}

\section{Roberto Valencia Arriaga \\ Santiago Capraro Rodríguez \\ Samuel Ortiz Velásquez*}

\section{RESUMEN}

El objetivo de este artículo es demostrar que la utilización de un modelo de crecimiento guiado por exportaciones en un régimen de metas de inflación ha resultado nocivo para el crecimiento económico en México, pues ha sido necesario aplicar un tipo de cambio sobrevaluado y contraer los salarios reales. La razón es que la dependencia de insumos importados del sector exportador impide que México use un tipo de cambio competitivo, como lo hicieron otros países con una estrategia similar, mientras que la contracción de los salarios comprime el mercado interno e inhibe el crecimiento desde adentro. Se estiman dos modelos tipo ARDL con los cuales se busca dar cuenta de esta hipótesis.

Palabras clave: crecimiento guiado por exportaciones, tipo de cambio, metas de inflación.

Clasificación JEL: F45, F31, E31

1 Este trabajo forma parte de la investigación realizada gracias al programa UNAM-PAPIIT número IA303318: "Metas de inflación y su impacto en el ecosistema de negocios mexicano", patrocinado por DGAPA-UNAM. Un primer acercamiento teórico del presente trabajo fue publicado en Issues in Business Management and Economics (2018), vol. 6 (1).

* Profesores de tiempo completo, Facultad de Economía, Universidad Nacional Autónoma de México, México. Correo electrónico: rova_35@yahoo.com.mx, santiago.capraro@gmail.com y samuelov@economia.unam.mx, respectivamente. 


\section{ABSTRACT \\ Export-led Growth and Inflation Targets in Mexico: a Bet Against Growth}

The objective of this article is to demonstrate that the use of an exportled growth model in an inflation-targeting regime is harmful for the economic growth in México because it has been necessary to use an overvalued exchange rate and to shrink real wages. The reason is that the dependence on imported inputs of exports hinder Mexico to use a competitive exchange rate like other countries that adopted a similar strategy. Besides, wage shrinkage has compressed the internal market inhibiting growth from within. Two models ARDL are estimated in order to prove this hypothesis.

Keywords: Export-led Growth, Exchange Rate, Inflation Targeting. JEL Classification: F45, F31, E31

\section{INTRODUCCIÓN}

Los problemas de crecimiento y la elevada inflación que México vivió durante la década de 1980 condujeron a los responsables de la política económica a realizar ajustes radicales. Entre los más importantes están haber adoptado un modelo de Crecimiento Guiado por Exportaciones (ELG, por sus siglas en inglés) y adherirse al Régimen de Metas de Inflación (RMI). Con el primero se buscaba reactivar el crecimiento, mientras que con el segundo, abatir la inflación.

La hipótesis de este trabajo es que la combinación de estas estrategias ha sido nociva para el crecimiento económico en México, pues eliminar la inflación ha traído menores tasas de crecimiento. Las razones se pueden resumir en tres puntos: primero, dado que las exportaciones mexicanas tienen un alto contenido importado, no ha sido posible utilizar un tipo de cambio competitivo, como lo hicieron los tigres asiáticos y otros países exitosos que adoptaron el ELG (Palley, 2012), pues esto tendría un impacto en la inflación. Segundo, en lugar de aumentar los salarios, como se esperaba, gracias a la apertura comercial (Salinas, 2000), éstos se contrajeron. De hecho, esto fue utilizado como ventaja competitiva para atraer inversiones extranjeras (Vázquez y Avendaño, 2012; Palley, 2012). Tercero, aunque el Banco de México afirma que 
el control de la inflación se dio por el anclaje de las expectativas de los agentes (Carstens, 2015), se ha documentado que la sobrevaluación del tipo de cambio y la caída en los salarios reales fueron la clave para lograrlo (Mantey, 2011; Esquivel y Razo, 2003; Perrotini y Vázquez, 2017).

Con este contexto, el objetivo de este artículo es demostrar que la utilización simultánea de un ELG y el RMI ha sido nocivo para el crecimiento económico en México. El trabajo se conforma de cinco apartados. En la segunda sección se encontrará una breve revisión de la literatura. En la tercera se expone un sencillo modelo macroeconómico, no ortodoxo, cuyo fin es formalizar la hipótesis del trabajo. En la cuarta se estiman dos modelos ARDL con el fin de conectar al ELG y RMI, y evaluar su impacto en el crecimiento. Finalmente, se presenta un breve apartado de conclusiones.

\section{REVISIÓN DE LA LITERATURA Y HECHOS ESTILIZADOS}

Después de la llamada crisis de la deuda, México dio un giro radical a su política económica con el objetivo principal de retomar la senda del crecimiento. De acuerdo con Salinas (2000), para lograrlo también era necesario reducir la inflación; en palabras del Banco de México "tanto las empresas como las familias se verían favorecidas por la ausencia de sorpresas inflacionarias, que pusieran en riesgo su patrimonio $\mathrm{y}$, por tanto, tendrían una mayor certidumbre para emprender planes y proyectos de largo plazo, lo que favorecería el ahorro y la inversión" ${ }^{2}$ (2004: 14).

Para lograr el primer fin, se adoptó un modelo de Crecimiento Guiado por Exportaciones (ELG), del cual se esperaba un impulso externo que arrastrara a la economía gracias a la dinámica del sector exportador, y un ímpetu interno, motivado por la mejora en los salarios, consecuencia de la convergencia salarial causada por la competencia internacional (Salinas, 2000).

\footnotetext{
2 Las cursivas son nuestras.
} 
En cuanto al segundo punto, se adoptó el Régimen Metas de Inflación (RMI), cuyo objetivo fue abatir el serio problema de inflación que se vivía desde años atrás.

Para comenzar con el ELG, debe decirse que antes que México se adhiriera, ya habían ocurrido algunas etapas: la primera inició después de la Segunda Guerra Mundial y concluyó alrededor de 1970. En esta fase, Alemania y Japón fueron los protagonistas, quienes aprovecharon el ímpetu de la reconstrucción de sus respectivas naciones. Una característica esencial de esta fase fue un Estado fuerte y la subvaluación del tipo de cambio (Palley, 2012).

La segunda inició en la década de 1980 con los llamados "tigres asiáticos". Hubo un impulso al desarrollo tecnológico guiado por un Estado fuerte. Además, se repite el uso de un tipo de cambio competitivo (Kokko, 2002; Palley, 2012).

En estas fases, además de las características anteriores, se pueden resaltar las siguientes: a) restricciones a la inversión extranjera directa; b) limitantes a la importación de algunos bienes clave, c) impulso a la educación orientada a la innovación (Blecker, 2000; Palley, 2012).

La intervención de México comenzó con la tercera etapa. Si bien es cierto que desde 1980 ya se participaba de una economía abierta, esto se potenció hasta la entrada en vigor del Tratado de Libre Comercio de América del Norte (TLCAN) en 1994.

GRÁFICA 1

TASA DE CRECIMIENTO ANUALIZADA DEL PIB (1993-2017)

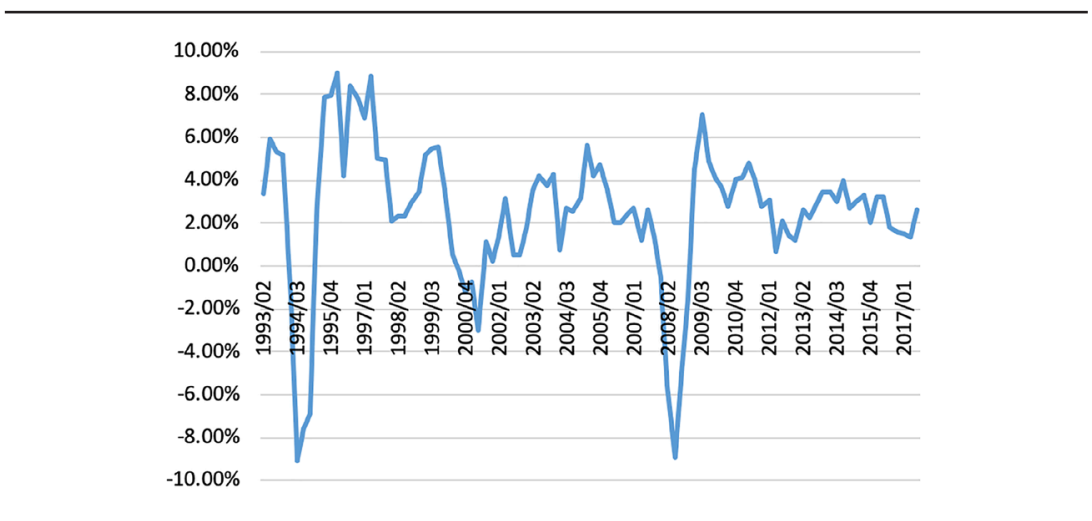

Fuente: elaborado con datos del Inegi (2020). 
En esta historia, México quedaría a la sombra de sus antecesores, pues sus resultados no serían iguales, ya que lejos de alcanzar una mayor tasa de crecimiento, disminuiría.

En la Gráfica 1 se observa que en la época de Zedillo, a pesar de la crisis, se alcanzó un crecimiento promedio de 3.39\%. Durante los gobiernos de Fox y Calderón, hasta antes de la gran crisis de 2008, la tasa media fue de $2.37 \%$, mientras que después de la crisis fue de 2.07 por ciento.

Lo anterior ha motivado diversas explicaciones. La mayoría coinciden en lo siguiente: la reducida participación del Estado permitió la apertura del mercado a grandes competidores internacionales; éstos convirtieron a México en una plataforma de exportación (Palley, 2012), reforzada por facilidades legales y fiscales (Moreno et al., 2005), para que llegaran inversiones extranjeras y aprovecharan la posición geográfica del país. Además, después de la crisis de 1994, los salarios reales en México cayeron drásticamente; si bien, tras la entrada en vigor el TLCAN, aumentó la productividad en el sector manufacturero, esto no se vio reflejado en los salarios (Polaski, 2003). Bajos salarios y cercanía

\section{GRÁFICA 2}

SALARIO REAL POR HORA EN DÓLARES EN LA INDUSTRIA MANUFACTURERA MEXICANA (A) Y CRECIMIENTO TRIMESTRAL DEL SALARIO BASE DE COTIZACIÓN REAL (B)

(A)

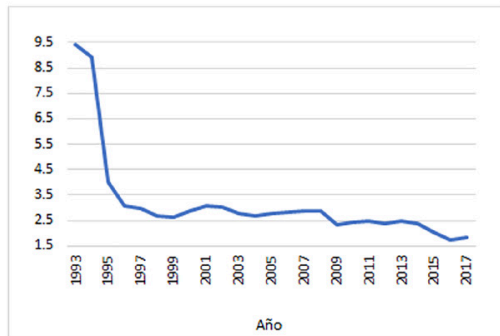

(B)

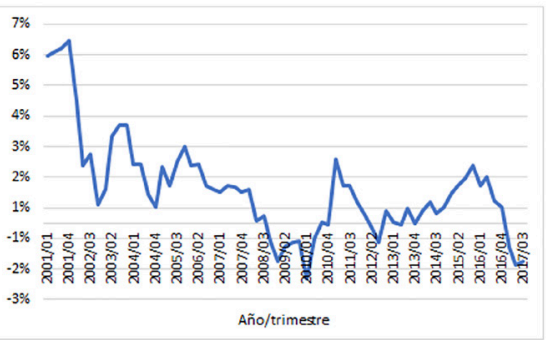

Fuente: elaborado con datos del Inegi (2020). 
con Estados Unidos fueron los principales atractivos del país que se ofrecían al exterior (Vázquez y Avendaño, 2012)

El panel A de la Gráfica 2 muestra una caída notable en el salario manufacturero. Al realizar la conversión en función del tipo de cambio, se observa que el salario de un trabajador en 2017 era de 272.25 pesos por jornada laboral en promedio, mientras que en 1993 se ganaban 231.77; es decir, el crecimiento ha sido de apenas $17.47 \%$ en 23 años. ${ }^{3}$ El panel $\mathrm{B}$ da cuenta del crecimiento del salario medio en el país. Este indicador tiene la bondad de incluir las prestaciones que un trabajador formal devenga. Aun así, la suerte de este indicador no dista de la gráfica previa.

Por otro lado, en la literatura económica es difícil encontrar un consenso sobre el efecto del tipo de cambio en el producto. Por ejemplo, mientras en Williamson (1990), Rodrik (1980) y Huerta (2006) se defiende la existencia de una relación directa entre ambas, en Krugman y Taylor (1978), López y Cruz (1999) y Blecker y Razmi (2009) se esgrime en favor de un efecto negativo. En este camino, se atribuye una parte importante al éxito del ELG el uso de un tipo de cambio competitivo. Evidencia de ello se encuentra en Palley (2012); Briseño (2013); Oreiro en Fiorito et al. (2011); Kan (2000); Koko (2002) y The World Bank (1993). En la Gráfica 3 se muestra que México no siguió este comportamiento.

GRÁFICA 3

MÉXICO: TIPO DE CAMBIO REAL, 1993-2017 (PESO/DÓLAR)

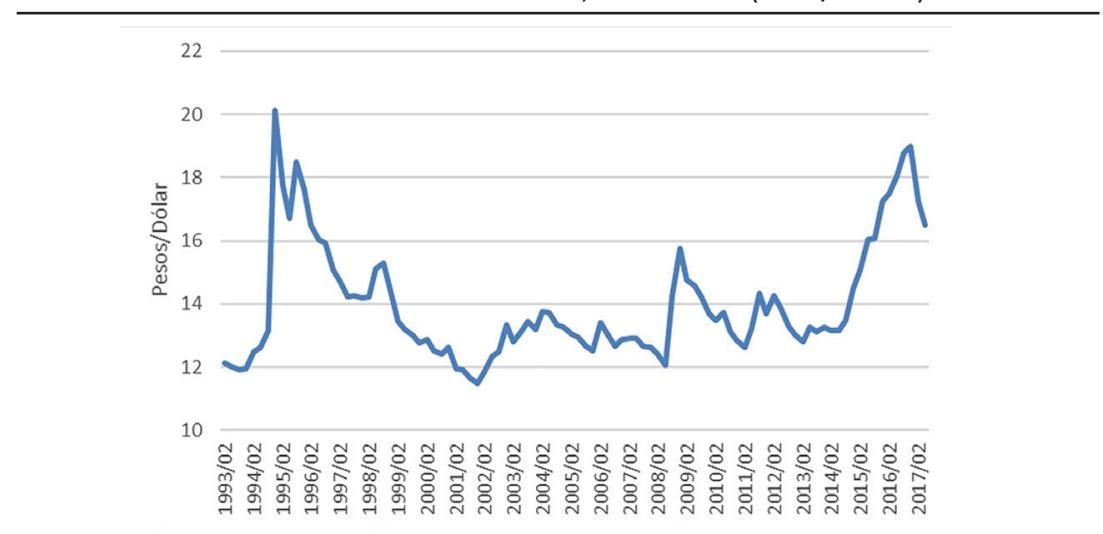

Fuente: elaborado con datos del Inegi (2020).

3 El cálculo se hizo tomando en cuenta un tipo de cambio de 3.1152 pesos por dólar en 1993, y 18.9066 en 2017 (Inegi, 2020). Además, se ha considerado una jornada laboral de 8 horas. 
La gráfica ilustra la sobrevaluación del tipo de cambio durante casi todo el periodo. Es importante anotar que, así como la contracción del salario real, la apreciación de la moneda no fue casual; ambas formaron parte de la estrategia para abatir la inflación (Mantey y López, 2010; Esquivel y Razo, 2003; Perrotini y Vázquez, 2017).

Al respecto, en 1999 México adoptó el RMI como estrategia para controlar la inflación. Las premisas eran: 1) hacer un anuncio explícito de una meta de inflación, la cual es de $3 \%$, con un margen de másmenos $1 \%$, y 2) un mecanismo de alta transparencia donde se informe constantemente sobre los actos del banco central. El fin era anclar las expectativas de los agentes dada su confianza en la institución (AguilarArgaez et al., 2014).

En el RMI se sostiene que un banco central puede inferir en el comportamiento de la inflación cuando ésta es producto de choques de demanda. Se asume que existe un producto potencial compatible con la meta de inflación, de modo que cuando el producto observado supera al potencial, se rebasa la meta de inflación (Arestis, 2009). Ante esto, un aumento en la tasa de interés real sería suficiente para frenar la demanda, luego vendría una caída en la inflación, consecuencia de un aumento temporal en el desempleo (Carlin y Soskice, 2006).

En el RMI se da poca importancia al tipo de cambio; sin embargo, en economías como la mexicana, la tasa de interés es un instrumento de control indirecto, dado que un incremento de ésta aprecia el tipo de cambio, lo que abarata los insumos importados y contiene el traspaso a los precios; en economías con alta dependencia a ello es complicado ignorarlo. Por tanto, el tipo de cambio se vuelve un objetivo intermedio para controlar la inflación. Evidencia de ello se muestra en Mantey (2011), Aizenman et al. (2008), Ibarra (2008) y Cruz et al. (2011).

Galindo y Ros (2006) llaman a esto "dominación externa", y se refieren a la dependencia de los precios internos del tipo de cambio. De acuerdo con los autores, el problema ocurre cuando una economía tiene una alta propensión al consumo de bienes importados. Una forma de medirlo es con el componente importado en las exportaciones, como se hace enseguida: 
GRÁFICA 4

INSUMOS IMPORTADOS EN LA PRODUCCIÓN DE BIENES DE EXPORTACIÓN (2007-2018)

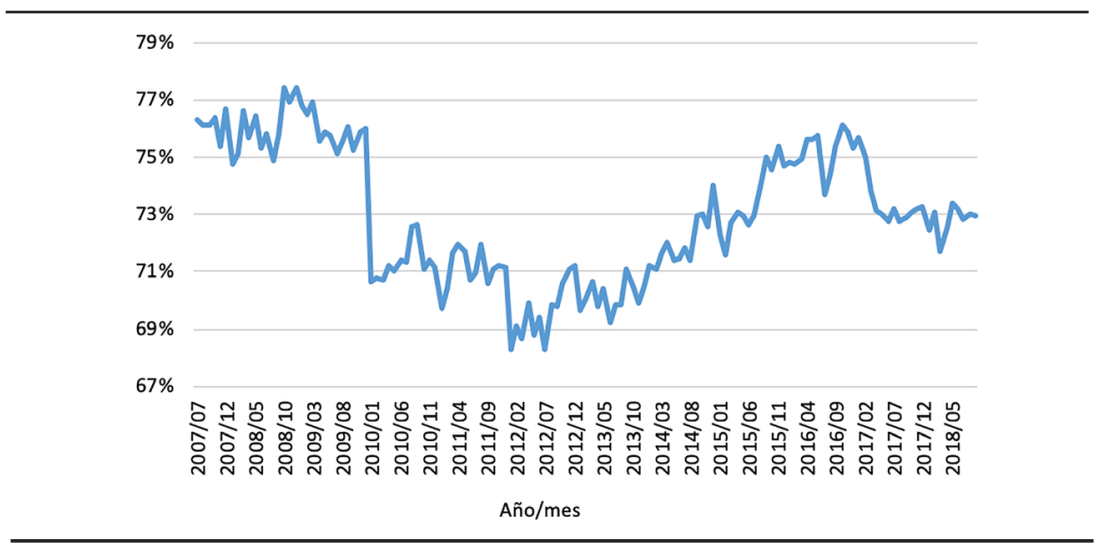

Fuente: elaborado con datos del Inegi (2020).

En la Gráfica 4 se aprecia que en septiembre de 2018, por cada dólar que se gastó en la producción de bienes de exportación, 73 centavos fueron pagados a proveedores extranjeros. Esto es una razón de que en México no ha funcionado el ELG, pues el efecto arrastre esperado por la mayor demanda de insumos y mano de obra internos requeridos para producir bienes de exportaciones es anulado una vez que el gasto es expulsado del país.

Lo anterior sugiere que esta estrategia de crecimiento contiene implícito un inhibidor externo del mismo, pues para exportar se requiere importar. Por esta razón no es posible usar un tipo de cambio competitivo; pues, aunque quizá ocurriría un aumento en las exportaciones, éstas vendrían acompañadas de más importaciones, pero más costosas. Dado que México no sólo es demandante de insumos extranjeros sino también de bienes finales, se pondría en peligro la meta de inflación. ${ }^{4}$

En suma, México tiene en el tipo de cambio un solo instrumento para dos objetivos: crecimiento e inflación. Ante la imposibilidad de lograr ambos, se ha tenido que determinar una prioridad: la estabilidad de precios, de modo que el papel del tipo de cambio ha sido contener el efecto traspaso, aunque existen posturas como las de Galván et al. (2019), Cortés (2013), Capistrán et al. (2012), Nogueira (2007) y

4 Entre 1994 y 2018, la tasa media de crecimiento anualizada de consumo de bienes importados fue de $8.15 \%$ (cálculo con datos del Inegi, 2020). 
Ramos et al. (2005) que argumentan que este efecto ha desaparecido. No obstante, a partir de la siguiente gráfica, se podría cuestionar esta conclusión:

\section{GRÁFICA 5}

TASA DE CRECIMIENTO ANUALIZADA DEL TIPO DE CAMBIO REAL E INFLACIÓN (1994-2017)

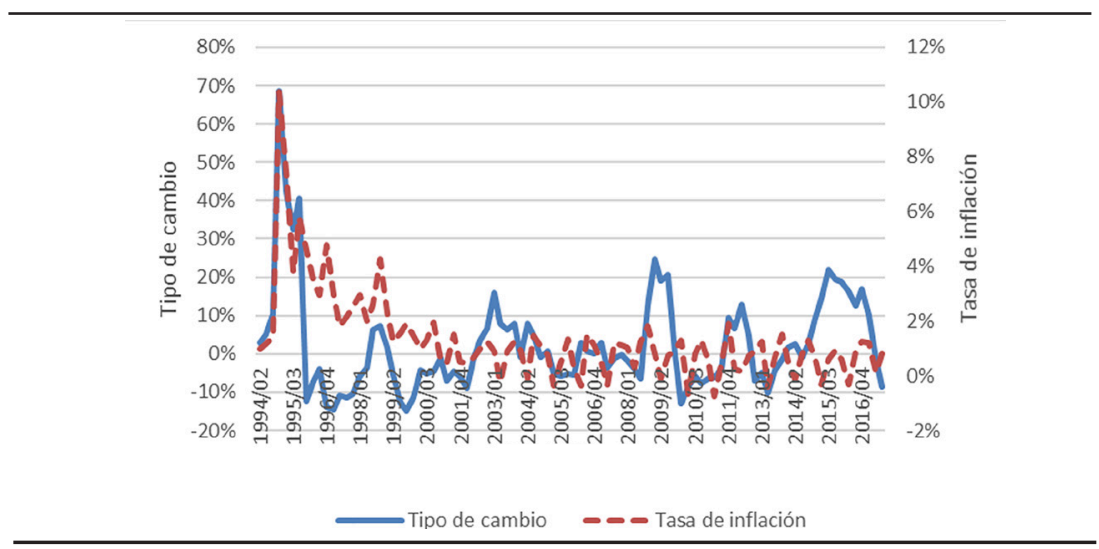

Fuente: elaborado con datos del Inegi (2020).

En la Gráfica 5 se observa una correlación directa entre la tendencia de ambas variables. De manera más formal, Aizenman et al. (2008), Cruz et al. (2011), Mantey (2011) y López y Valencia (2018) demuestran que el efecto traspaso continúa en México. En particular, en el último caso se señala que ha desaparecido el traspaso inmediato, pues los empresarios parecen detectar que en un mercado interno contraído -como ha ocurrido en México por la contracción salarial- el aumento en los precios podría disminuir la demanda, así que ellos preferirían sacrificar temporalmente la tasa de ganancia a cambio de permanecer en el mercado. Una razón más es que aquellos que tienen inventarios no requieren comprar a nuevos costos, por lo que pueden mantener sus precios basados en costos antiguos; sin embargo, una vez agotados los inventarios, si el tipo de cambio continúa depreciado, terminarán ajustando sus precios (Valencia y Hernández, 2018).

Otro hecho importante que ayudó a desinflar a la economía fue la contracción salarial que, como se señaló anteriormente, también fue 
utilizada por México como atractivo para llamar inversiones extranjeras. En este sentido, Seccareccia y Lavoie (2010) muestran cómo en Canadá la caída en los salarios reales ayudó a contener la inflación. Evidencia similar la aportan Esquivel y Razo (2003), Perrotini y Vázquez (2017) y López y Valencia (2018) para el caso mexicano. De sus resultados se desprende que en México la inflación no es un problema de demanda, sino de costos y un conflicto distributivo, como defienden Argitis y Pitelis (2001). Desde luego, la caída en los salarios se ha reflejado en la contracción del mercado interno, por lo que el RMI contiene un inhibidor interno del crecimiento.

\section{UN MODELO TEÓRICO ALTERNATIVO}

En esta sección se construirá un sencillo modelo macroeconómico. La idea no es sustituir los existentes en la literatura económica, como el de tres ecuaciones de Carlin y Soskice (2006), la crítica heterodoxa al mismo, de Setterfield (2006), o el de Arestis (2009); únicamente se busca formalizar las ideas de la sección anterior con el fin de proveer de insumos para la estimación de dos modelos econométricos.

Durante la exposición haremos algunos supuestos sobre los comportamientos esperados de cada variable dependiente respecto de las exógenas, con base en la evidencia empírica de la sección anterior y otros trabajos. El modelo propuesto es el siguiente:

$$
\begin{aligned}
& \mathrm{J}=\mathrm{J}\left(\theta, \mathrm{Y}_{\mathrm{t}-1}, \mathrm{Y}^{*}\right), \quad \mathrm{J}_{\theta}, \mathrm{J}_{\mathrm{Y}^{*}}>0 ; \mathrm{J}_{\mathrm{Yt}-1}<0 \\
& \mathrm{C}=\mathrm{C}(\mathrm{w}), \quad \mathrm{C}_{\mathrm{w}}>0 \\
& \mathrm{I}=\mathrm{I}\left(\mathrm{Y}_{\mathrm{t}-1}\right), \quad \mathrm{I}_{\mathrm{Yt}-1}>0 \\
& \mathrm{Y}=\mathrm{C}+\mathrm{I}+\mathrm{G}+\mathrm{J}, \mathrm{J}=\mathrm{X}-\mathrm{M} \\
& \pi=\pi(\mathrm{w}, \theta, \mathrm{q}, \mathrm{r}), \pi_{\mathrm{w}}, \pi_{\theta}, \pi_{\mathrm{r}}>0 ; \pi_{\mathrm{q}}<0
\end{aligned}
$$


Donde: J=Saldo neto de la balanza comercial; $\theta=$ Tipo de cambio real; $\mathrm{Y}^{*}=\mathrm{PIB}$ de Estados Unidos; $\mathrm{Y}_{\mathrm{t}-1}=\mathrm{PIB}$ de México rezagado; $\mathrm{C}=$ Consumo interno; $\mathrm{w}=$ Salarios reales; $\pi=$ tasa de inflación; $\mathrm{I}=$ Inversión; $\mathrm{G}=\mathrm{Gasto}$ público; $\mathrm{X}=$ =xportaciones; $\mathrm{M}=$ importaciones; $\mathrm{q}=$ productividad, $\mathrm{y} \mathrm{r}=$ tasa de interés.

En (1) se define el saldo neto de la balanza comercial en función del tipo de cambio real $(\theta)$ del PIB de Estados Unidos ( $\mathrm{Y}^{*}$ ) y del PIB mexicano rezagado $\left(\mathrm{Y}_{\mathrm{t}-1}\right)$. El resultado esperado de la derivada parcial de $J$ respecto al tipo de cambio real $(\theta)$ se expresa de la forma $J_{\theta}>0$, lo cual implica que hay una respuesta con signo positivo de la balanza comercial a los movimientos del tipo de cambio real; es decir, ante una depreciación (aumento de $\theta$ ), las exportaciones aumentarán y las importaciones disminuirán. Por el contrario, una apreciación reduciría el monto de (X) y alentaría (M). Esta lógica se basa en el supuesto de que se cumple la condición Marshall-Lerner (M-L), lo cual parece haber ocurrido en aquellos países exitosos que adoptaron el ELG, como se documentó en la sección anterior. Según los resultados de Galindo y Guerrero (1997), y Arriaga y Landa (2016), en México se cumple M-L, así que asumiremos que $\mathrm{X}$ y $\mathrm{M}$ son elásticas a $\theta$. Con base en esto, se esperaría que una depreciación tuviera efectos positivos en el producto. Sin embargo, en México esto no ocurre. Más bien se comporta de la siguiente forma:

$$
\mathrm{Y}_{\theta} \approx 0
$$

En (1.1) se indica que la derivada parcial del producto respecto al tipo de cambio real es cercana a 0 , debido a que, aun aceptando que una depreciación mejore el saldo neto de la balanza comercial, la dependencia de las exportaciones mexicanas de insumos importados (Vázquez y Avendaño, 2012) provocaría que el efecto positivo sobre el producto fuera anulado, ya que parte importante de la riqueza sería derramada en el exterior.

Como se ilustró en la Gráfica 4, tres cuartas partes del gasto realizado para producir bienes de exportación se efectúa en el extranjero, lo cual es producto de una alta concentración del comercio internacional en unas cuantas industrias, además del intercambio que se realiza de tipo intraindustria o incluso intrafirma, como se defiende en Dussel y 
Ortiz (2016) y Calderón y Hernández (2016), respectivamente. Todo esto contribuye a que los beneficios esperados de la dinámica exportadora no se expandan a toda la economía.

Además, una depreciación encarecería no sólo las materias primas importadas, sino también los bienes finales importados; elevar los costos de producción y de ventas disminuiría la ganancia de los empresarios, quienes defenderían su participación en el producto con el incremento de sus precios, si bien no de manera inmediata, como se discutió en la sección anterior, sí en el largo plazo. La consecuencia sería un aumento en la inflación y, de acuerdo con la ecuación (2), caería el consumo, luego de la contracción en los salarios reales. Posteriormente vendría una caída en el crecimiento del producto.

En suma, el hipotético efecto expansivo de un aumento en las exportaciones podría ser minado por el mayor costo de bienes importados y por la caída en el consumo.

De (1) se desprende también que la balanza comercial es sensible a la elasticidad ingreso de las exportaciones $\left(\mathrm{J}_{\mathrm{Y}^{*}}>0\right)$ y de las importaciones $\left(\mathrm{J}_{\mathrm{Yt}-1}<0\right)$. Es decir, ante un mayor crecimiento de la economía estadounidense, el saldo en (J) sería favorable debido a una mayor demanda de nuestras exportaciones (Galindo y Guerrero, 1997; Arriaga y Landa, 2016). Este resultado es producto de la sincronización de los ciclos económicos ${ }^{5}$ entre las industrias mexicana y estadounidense, como se argumenta en Rodríguez et al. (2015), quienes presentan evidencia de un ciclo común de corto plazo entre ambos países. Esto lo confirman Calderón y Hernández (2016). Por su parte, Dussel y Ortiz (2016) señalan que el comportamiento cíclico entre ambas naciones ocurre a nivel industria. Echenique y López (2020) abonan señalando que por cada punto porcentual que crece el índice de producción manufacturera de Estados Unidos, el PIB manufacturero de México aumenta 1.53 por ciento.

En (1) también se ve que un crecimiento "excesivo" del producto nacional de hoy podría llevar a una contracción de la balanza comercial de futuros periodos. Es decir:

$$
1>Y_{Y^{*}}>0
$$

5 Agradecemos a un árbitro anónimo por esta sugerencia. 
En (1.2) se plantea que el crecimiento económico es sensible al crecimiento de nuestro principal socio comercial, como reportan Caballero y López (2013); sin embargo, hay un límite, pues se frenará en el momento en que las importaciones aumenten como consecuencia de una tasa de crecimiento nacional "excesiva", es decir, superior a aquella compatible con el exterior (Thirlwall, 1979). A esto se le conoce como la restricción externa al crecimiento y ha sido documentada para México por Arenas et al. (2015); Clavijo y Ros (2015); Perrotini (2002), y López y Cruz (1999).

Este efecto se puede formalizar de la siguiente manera:

$$
\mathrm{Y}_{\mathrm{Yt}-1}<0, \leftrightarrow \Delta \mathrm{Y} \rightarrow \Delta \mathrm{X}<\Delta \mathrm{M}
$$

En (1.3) se muestra que el producto del periodo corriente se verá influido negativamente por la evolución del pasado sí, y sólo sí, el crecimiento en el producto corriente conlleva que el aumento en las importaciones sea mayor al crecimiento en las exportaciones. Así, mientras no se cumpla (1.3), un aumento en $\mathrm{Y}^{*}$ hará que la economía nacional crezca gracias a un mayor dinamismo de nuestras exportaciones.

Probablemente el lector se cuestione por qué en este caso un aumento en las exportaciones no deje los mismos resultados que el aumento en las exportaciones ocasionado por una depreciación. La diferencia estriba en que, con una moneda depreciada, el costo de los insumos importados aumenta, por lo que tendría un efecto contraccionista en el corto plazo, como apuntan Krugman y Taylor (1978), ya que, como se explicó, se elevaría la tasa de inflación. Esto reduciría el salario real, después caería el consumo, lo que ocasionaría un efecto sustitución, donde el mayor gasto del exterior (aumento en las exportaciones) podría ser disipado por una contracción en el gasto interno (Carlin y Soskice, 2006). El efecto continuaría, puesto que la caída en el salario real y el consumo llevarían a un menor nivel de producto, causando una contracción en la inversión en periodos siguientes. El resultado final dependerá de qué variables sean más afectadas; la depreciación podría alentar a las exportaciones, pero también podría disminuir el consumo y la inversión.

Contrariamente, si el incremento se da por una expansión del producto extranjero, el aumento en las exportaciones también acarrearía 
más importaciones, pero sin cambios en los precios relativos, de suerte que no habría efectos de segundo orden. Así que habría un saldo positivo en balanza comercial; el salario real se mantendría constante, al igual que el consumo. El resultado final sería favorable para el producto nacional, toda vez que la inversión aumentaría el siguiente periodo. Dicho de otro modo, el efecto de la elasticidad ingreso de nuestras exportaciones es mayor que el efecto de la elasticidad precio.

Formalmente:

$$
\mathrm{Y}=\mathrm{Y}\left(\theta, \mathrm{Y}^{*}\right) \text {, donde } \mathrm{Y}_{\theta}<\mathrm{Y}_{\mathrm{Y}^{*}}
$$

En (2) se define que el consumo interno (C) depende de forma directa del salario real $(w)$; desde luego, esto implica que el salario nominal

tiene un efecto positivo sobre $(\mathrm{C})$, mientras que la inflación $(\pi)$ influye de forma inversa.

Así que a partir de (2) y (4) se deduce que:

$$
\mathrm{Y}_{\mathrm{w}}>0 \quad(2.1) \text { y } \quad \mathrm{Y}_{\pi}<0
$$

Es decir, dado que supondremos que los asalariados no ahorran (Kalecki, 1954), los aumentos en el salario deberían tener un efecto multiplicador en el producto, mientras existan recursos ociosos (Caballero y López, 2013). Por el contrario, en (2.2) se muestra que los aumentos en la tasa de inflación contraen el producto. La razón es que una mayor inflación mina el salario real, esto reduce el consumo y, por ende, el producto y la inversión.

En (3) se define a la inversión en función del desempeño del PIB de periodos atrás. Esto se apoya en la tesis de Kalecki (1954), donde se expone que los empresarios toman sus decisiones de inversión en función de los resultados del pasado, por lo que, si en periodos previos hubo una expansión de la demanda, decidirán invertir más en el periodo actual.

En (4) se expresa la identidad del PIB. Dado que el RMI ha relegado la participación de la política fiscal, supondremos que $\mathrm{G}$ es constante, por lo que (4) se expresaría así:

$$
\mathrm{Y}=\mathrm{C}+\mathrm{I}+\mathrm{J}
$$


Con base en lo anterior, tenemos que el crecimiento del PIB dependerá de:

$$
\mathrm{Y}=\mathrm{Y}\left(\theta, \mathrm{w}, \pi, \mathrm{Y}^{*}\right) \text {, con } \mathrm{Y}_{\theta} \approx 0<\mathrm{Y}_{\mathrm{Y}^{*}}<1 ; \mathrm{Y}_{\mathrm{w}}>0 ; \mathrm{Y}_{\pi}<0
$$

Es decir, se espera que el efecto del tipo de cambio real sobre el producto sea casi nulo; el crecimiento del producto del extranjero influiría positivamente en la economía nacional, aunque limitado por la restricción externa al crecimiento; la evolución de los salarios reales tendrá un impulso positivo. Finalmente, el efecto de la inflación será negativo.

En (5) se plantea que la tasa de inflación $(\pi)$ depende de forma positiva de (w) y de $(\theta)$, además de la tasa de interés (r), y de forma inversa, de la productividad (q). Estas variables tradicionalmente las sigue el Banco de México, aunque también suele considerar el PIB y el PIB potencial. ${ }^{6}$ No obstante, siendo consistentes con un marco heterodoxo, nos adherimos a las ideas originales de Keynes (1936) y Kalecki (1954), quienes afirman que un aumento en el producto sólo tendrá efectos en la inflación hasta que dejen de existir recursos ociosos.

La inclusión de (w) y (q) es compatible con los resultados de López y Valencia (2018), quienes destacan que la variable que tiene efectos sobre $(\pi)$ no es propiamente $(w)$, sino el costo del trabajo $(u)$, el cual se define de la siguiente manera:

$$
u=\frac{w}{q}
$$

De (5.1) se desprende que la inflación estará influida por la distribución del ingreso (Setterfield, 2006), pues un aumento en (w) redistribuye el ingreso en contra de los empresarios, ocasionando que busquen defenderse subiendo sus precios (Kaldor, 1980; Panico, 1988). Cuando (q) aumenta, hay un incremento en la rentabilidad del factor trabajo. Esto se traduce en mayores beneficios para la empresa, por lo que minan las razones para elevar los precios debido al excedente, del que difícilmente se apropian los trabajadores, pero del que se adueñan los empresarios para mejorar su participación en el producto (Capraro, 2018). El efecto esperado de $(\theta)$ sobre $(\pi)$ ha sido ampliamente discutido anteriormente.

6 Véanse los informes anuales del Banco de México. 
La inclusión de la (r) es consistente con el RMI (Carlin y Soskice, 2006), ya que refleja el papel de la política monetaria. Nuestra diferencia estriba en que nosotros esperamos $\pi_{\mathrm{r}}>0$, ad hoc con un marco que incluya la distribución del ingreso, como exponen Kaldor (1980) y Argitis y Piteles (2001), quienes observan en (r) un costo. Así que, si el banco central sube su tasa, la banca comercial también lo hará, pero en mayor medida, lo que provocará una redistribución del ingreso en favor de ellos. La reacción de los empresarios sería buscar trasladar dicho costo a los trabajadores vía inflación.

\section{DOS MODELOS ARDL}

En esta sección mostraremos evidencia de lo discutido hasta ahora con la estimación de dos modelos tipo ARDL. El primero busca medir el impacto de las variables relevantes en un modelo ELG, por lo que partiremos de (4.2). Para el segundo nos apoyaremos de (5), con el fin de detectar la conexión del RMI con el crecimiento económico.

\subsection{Metodología y estimación del modelo}

Los modelos Autoregresivos con Rezagos Distributivos (ARDL) se basan en la metodología propuesta por Pesaran et al. (2001). Su ventaja es que se pueden incluir series de tipo I (0) e I (1), lo cual no permiten otras técnicas, como Johansen o Engle y Granger. Es condición necesaria para obtener resultados confiables no trabajar con series tipo I(2). La técnica permite también estimar prácticamente al mismo tiempo el modelo de largo plazo y el de corto plazo (Ibarra, 2011; Laurenceson y Chai, 2003).

De (4.2) y (5) se desprenden ambos modelos, expresados en (6) y (7) de forma ampliada:

Todas las variables han sido definidas en la sección anterior. El símbolo $\Delta$ denota la tasa de crecimiento de la variable en cuestión, mientras $\varepsilon$ se refiere al término de error.

$$
\begin{aligned}
& \Delta y=\sum_{i=1}^{4} \alpha_{i} \Delta y_{t-i}+\sum_{i=0}^{4} \beta_{i} \Delta y^{*}{ }_{t-i}+\sum_{i=o}^{4} \mathrm{c}_{i} \theta_{t-i}+\sum_{i=o}^{4} \lambda_{i} \pi_{t-i} \sum_{i=o}^{4} \varepsilon_{i} \mathrm{w}_{t-i}+\varphi_{1} \Delta y_{t-1}+ \\
& \varphi_{2} \Delta y_{t-1}^{*}+\varphi_{3} \theta_{t-1}+\varphi_{4} \pi_{t-1}+\varphi_{5} \mathrm{w}_{t-1}+\varepsilon
\end{aligned}
$$




$$
\begin{aligned}
& \pi=\sum_{i=1}^{4} a_{i} \pi_{t-i}+\sum_{i=0}^{4} b_{i} w_{t-i}+\sum_{i=o}^{4} \mathrm{~d}_{i} \Delta \theta_{t-i}+\sum_{i=o}^{4} n_{i} \mathrm{r}_{t-i}+\sum_{i=o}^{4} k_{i} \mathrm{q}_{t-i}+\mu_{1} \pi_{t-1}+ \\
& \mu_{2} w_{t-i}+\mu_{3} \Delta \theta_{t-1}+\mu_{4} \mathrm{r}_{t-1}+\mu_{5} \mathrm{q}_{t-1}+\varepsilon
\end{aligned}
$$

Las variables están expresadas en logaritmos, por lo que el resultado puede ser interpretado como una elasticidad. Para (r) se usaron cetes a 28 días. El periodo de estimación va del primer trimestre de 2000 al tercer trimestre de 2017. Se trabaja con datos trimestrales extraídos de INEGI y del Bureau of Economics Analysis (BEA).

Las pruebas de raíces unitarias se muestran en la Tabla 1. De ella se desprende que (r) es I(1), mientras no hay evidencia contundente de que (p), (w) y (q) sean estacionarias en niveles. El resto son claramente I(0). Por esta razón, se confirma que la metodología utilizada es adecuada, pues de haber sido todas I(1), lo recomendable hubiera sido estimar un VEC usando la técnica de Johansen, mientras que de ser I(0), la sugerencia hubiera sido un VAR (Pesaran et al., 2001; Ibarra, 2011; Pérez, 2008; Loría, 2007).

Los resultados de la estimación de ambos modelos se muestran en la Tabla 2.

TABLA 1

PRUEBAS DE RAÍCES UNITARIAS

\begin{tabular}{cccccccccc}
\hline Variable & Intercepto & $\begin{array}{c}\text { ADF } \\
\text { Tendencia e } \\
\text { intercepto }\end{array}$ & Nada & Intercepto & $\begin{array}{c}\text { Pendencia e } \\
\text { intercepto }\end{array}$ & Nada & Intercepto & $\begin{array}{c}\text { Kendencia e } \\
\text { intercepto }\end{array}$ \\
\hline y & 0.0144 & 0.0487 & 0.0089 & 0.0001 & 0.0001 & 0.0000 & 0.106573 & 0.091603 \\
\hline Dy & 0.0000 & 0.0001 & 0.0000 & 0.0001 & 0.0001 & 0.0000 & 0.059972 & 0.059427 \\
\hline$y^{*}$ & 0.0000 & 0.0002 & 0.0331 & 0.0000 & 0.0002 & 0.0175 & 0.185639 & 0.081316 \\
\hline Dy* & 0.0000 & 0.0000 & 0.0000 & 0.0001 & 0.0001 & 0.0000 & 0.125933 & 0.119871 \\
\hline$\pi$ & 0.0162 & 0.0333 & 0.2409 & 0.0000 & 0.0000 & 0.0000 & 0.500000 & 0.136323 \\
\hline D $\pi$ & 0.0000 & 0.0001 & 0.0000 & 0.0001 & 0.0001 & 0.0000 & 0.102970 & 0.090778 \\
\hline$\theta$ & 0.0000 & 0.0000 & 0.0000 & 0.0000 & 0.0000 & 0.0000 & 0.075134 & 0.039089 \\
\hline D $\theta$ & 0.0000 & 0.0000 & 0.0000 & 0.0001 & 0.0001 & 0.0000 & 0.069813 & 0.060509 \\
\hline$w$ & 0.0643 & 0.1018 & 0.1258 & 0.0000 & 0.0000 & 0.0000 & 0.463051 & 0.105757 \\
\hline Dw & 0.0530 & 0.3553 & 0.0058 & 0.0001 & 0.0001 & 0.0000 & 0.113478 & 0.113554 \\
\hline$q$ & 0.9979 & 0.4807 & 0.6421 & 0.0005 & 0.0000 & 0.0000 & 0.932200 & 0.289200 \\
\hline Dq & 0.0021 & 0.0004 & 0.0003 & 0.0001 & 0.0001 & 0.0000 & 0.128100 & 0.072200 \\
\hline$r$ & 0.3198 & 0.8940 & 0.3180 & 0.2844 & 0.8036 & 0.3204 & 0.799400 & 0.097200 \\
\hline Dr & 0.0000 & 0.0000 & 0.0000 & 0.0000 & 0.0000 & 0.0000 & 0.206200 & 0.071600 \\
\hline
\end{tabular}

Para las pruebas ADF y PP se presentan las probabilidades. Los valores críticos de referencia para KPSS son 0.4630 co intercepto y 0.1460 con tendencia.

La letra $D$ se refiere a la primera diferencia de la variable en cuestión

Fuente: elaboración propia. 
Primeramente, debe destacarse el valor de la prueba de Wald, la cual es una prueba $\mathrm{F}$, cuyo resultado debe ser contrastado con los límites

TABLA 2

RESULTADOS DEL MODELO

\begin{tabular}{|c|c|c|c|c|c|c|c|c|c|}
\hline \multicolumn{5}{|c|}{ Determinantes del producto } & \multicolumn{5}{|c|}{ Determinantes de la inflación } \\
\hline Variable & Coeficiente $\mid$ & Error estándar & Estadístico $t$ & Probabilidad & Variable & Coeficiente & Error estándar & Estadístico $\mathrm{t}$ & Probabilidad \\
\hline$\Delta \mathrm{y}(-1)$ & -2.5827 & 0.2178 & -11.8593 & 0.0000 & $\pi(-1)$ & -1.1954 & \begin{tabular}{|l|}
0.1041 \\
\end{tabular} & -11.4882 & 0.0000 \\
\hline$\Delta \mathrm{y}^{*}(-1)$ & 0.6534 & 0.1530 & 4.2702 & 0.0001 & $\mathrm{w}(-1)$ & 1.0183 & 0.4730 & 2.1526 & 0.0363 \\
\hline$\theta(-1)$ & -0.0142 & 0.0067 & -2.1151 & 0.0396 & $\Delta \theta(-1)$ & 0.0098 & 0.0049 & 2.0165 & 0.0492 \\
\hline$\pi(-1)$ & -0.8324 & 0.1372 & -6.0671 & 0.0000 & $\mathrm{r}(-1)$ & 0.2488 & 0.0481 & 5.1670 & 0.0000 \\
\hline $\mathrm{w}(-1)$ & 0.2061 & 0.0678 & 3.0403 & 0.0038 & $\mathrm{q}(-1)$ & -1.2451 & 0.4449 & -2.7984 & 0.0073 \\
\hline Vyt -1 & -0.8008 & 0.2573 & -3.1122 & 0.0029 & $\mathrm{~V} \pi \mathrm{t}-1$ & -0.8399 & 0.1916 & -4.3838 & 0.0000 \\
\hline \multicolumn{5}{|c|}{ Estadísticos del Modelo* } & \multicolumn{5}{|c|}{ Estadísticos del Modelo* } \\
\hline \multicolumn{2}{|c|}{\begin{tabular}{|l|l} 
Largo plazo & \\
\end{tabular}} & \multicolumn{3}{|c|}{\begin{tabular}{|l} 
Corto plazo \\
\end{tabular}} & \multicolumn{2}{|c|}{\begin{tabular}{|l|l|} 
Largo plazo & \\
\end{tabular}} & \multicolumn{3}{|c|}{\begin{tabular}{|l} 
Corto plazo \\
\end{tabular}} \\
\hline R squared & 0.98355 & R squa & & 0.938339 & R squared & 0.8927 & R squa & & 0.8195 \\
\hline Durbin-Watson & 1.930736 & Durbin-W & Vatson & 1.841021 & Durbin-Watson & 2.2320 & Durbin-W & Vatson & 1.9034 \\
\hline \begin{tabular}{|l|} 
Jarque Bera \\
\end{tabular} & 0.7446 & Jarque $\mathrm{I}$ & Bera & 0.489361 & Jarque Bera & 0.8385 & Jarque 1 & Bera & 0.8962 \\
\hline LM (4) test & 0.858 & LM (4) & test & 0.1732 & LM (4) test & 0.3478 & LM (4) & test & 0.0507 \\
\hline B-P-G & 0.6002 & B-P- & & 0.0994 & B-P-G & 0.2382 & Breusch-Paga & an-Godfrey & 0.0765 \\
\hline Ramsey & 0.3757 & Rams & & 0.9839 & Ramsey & 0.7798 & Rams & & 0.9148 \\
\hline Wald & 40.7416 & & & & Wald & 29.4500 & & & \\
\hline \multicolumn{10}{|c|}{ Límites de Pesarán, Shin, Smith (2001) } \\
\hline Inferior & \multicolumn{9}{|c|}{2.26} \\
\hline Superior & \multicolumn{9}{|c|}{3.48} \\
\hline \multicolumn{5}{|c|}{ Vy t-1: Vector corrector de errores } & \multicolumn{5}{|c|}{$\mathrm{V} \pi \mathrm{t}-1:$ Vector corrector de errores } \\
\hline \multicolumn{10}{|c|}{ "Para las pruebas Jarque Bera, LM, B-P-G (Breusch-Pagan-Godfrey) y Ramsey, se reporta la probabilidad. } \\
\hline \multicolumn{5}{|c|}{$\begin{array}{l}\text { La prueba de Wald tiene esta Ho: parámetros }(y)=\left(y^{*}\right)=(\theta)=(\pi)= \\
(\mathrm{w})=0\end{array}$} & \multicolumn{5}{|c|}{ La prueba de Wald tiene esta Ho: parámetros $(\pi)=(\theta)=(\mathrm{w})=(\mathrm{r})=(\mathrm{q})=0$} \\
\hline
\end{tabular}

Fuente: elaboración propia con base en los resultados de las estimaciones.

propuestos por Pesarán et al. (2001), y de ser mayor, se aceptará que las variables cointegran. Para el modelo determinantes del producto el resultado es 40.74, y para el de determinantes de la inflación, 29.45. En ambos casos es superior a 3.48 (límite superior propuesto por los autores), por lo tanto, se acepta la hipótesis de cointregación. 


\section{GRÁFICA 6}

PRUEBA CUSUM

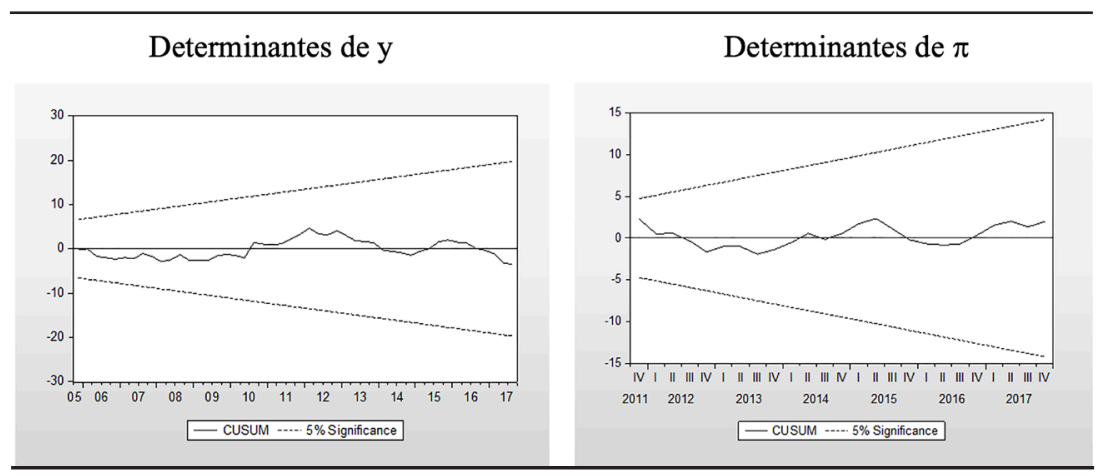

Fuente: elaboración propia con base en los resultados del modelo.

\section{GRÁFICA 7}

PRUEBA CUSUM Q

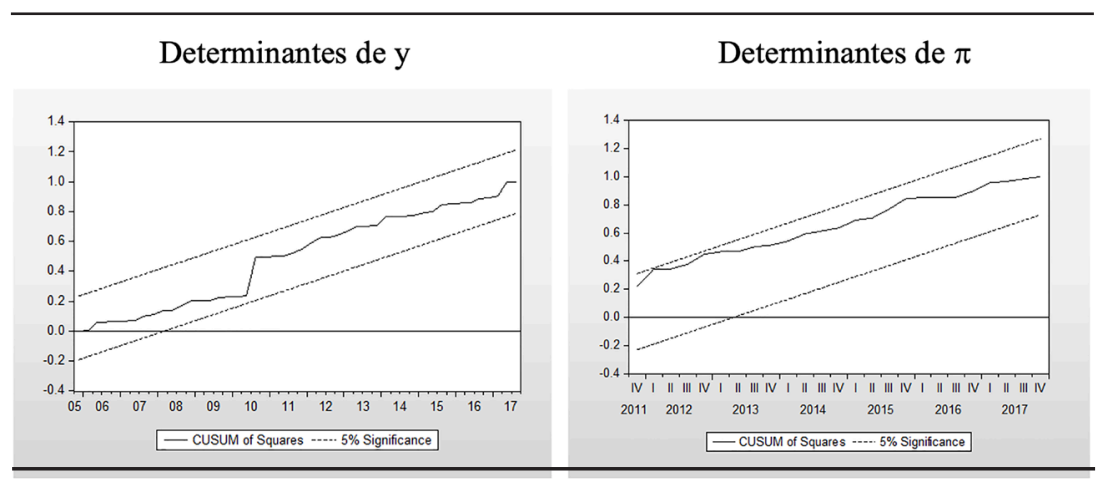

Fuente: elaboración propia con base en los resultados del modelo.

Las gráficas 6 y 7 muestran que ambos modelos no sufren de cambio estructural. Con los resultados de la Tabla 2 y siguiendo a Ibarra (2011) es posible calcular los multiplicadores de largo plazo:

$$
\begin{array}{lll}
\Delta y^{*}=-(\phi 2 / \phi 1)=0.2529 & (7) & w=-(\mu 2 / \mu 1)=0.8517 \\
\theta=-(\phi 3 / \phi 1)=-0.0054 & (8) & \Delta \theta=-(\mu 3 / \mu 1)=0.0082 \\
\pi=-(\phi 4 / \phi 1)=-0.3223 & (9) & r=-(\mu 4 / \mu 1)=0.2080 \\
w=-(\phi 5 / \phi 1)=0.0797 & (10) & q=-(\mu 5 / \mu 1)=-1.0415
\end{array}
$$


Donde $\phi 1, \phi 2, \phi 3, \phi 4$ y $\phi 5$, son los coeficientes de y(-1), $\mathrm{y}^{*}(-1), \theta(-1)$, $\pi(-1)$ y w(-1), respectivamente, y $\mu 1, \mu 2, \mu 3, \mu 4$ у $\mu 5$, de $\pi(-1)$, w(-1), $\Delta \theta(-1), \mathrm{r}(-1)$ y q(-1) correspondientemente, todos reportados en la Tabla 2. En la Tabla 2 también se reportan los resultados de los modelos correctores de errores. Para el caso del producto $\left(\mathrm{V}_{\mathrm{yt}-1}\right)$ es de 0.8008 , mientras que para la inflación $\left(\mathrm{V}_{\pi t-1}\right), 0.8399$. Los datos muestran que mientras el producto converge a su equilibrio a una velocidad de $80.08 \%$, la inflación lo hace a una velocidad de 83.99 por ciento.

\subsection{Discusión de resultados}

De acuerdo con (7) y (8), se valida el comportamiento esperado de las derivadas parciales planteadas en (1.1) y (1.2). Sin embargo, resalta que una depreciación no tiene un efecto positivo sobre el producto, sino que lo contrae. Aunque estadísticamente este resultado no es distinto de 0, siendo rigurosos, se validaría la hipótesis de Krugman y Taylor (1978). También esto es compatible con López y Cruz (1999) y Blecker y Razmi (2010).

El resultado del multiplicador en (8) no evidencia que no se cumpla la condición Marshall-Lerner para México, en realidad tampoco es argumento de lo contrario, lo único claro es que usar un tipo de cambio competitivo no tiene efectos positivos en la tasa de crecimiento del producto por las razones ya ampliamente discutidas en secciones previas.

También se observa la correlación positiva entre el crecimiento de Estados Unidos y México, de suerte que un crecimiento en $1 \%$ de ellos causa un incremento de $0.25 \%$ en México; el efecto pequeño se puede deber a que el gasto no se queda en el país, consecuencia de lo explicado previamente. En suma, los resultados ratifican que $\mathrm{Y}_{\mathrm{y}^{*}}>\mathrm{Y}_{\theta}$.

De (9) se desprende que hay una relación inversa entre producto e inflación. Evidencia sobre esta relación se puede encontrar en estudios de panel hechos por Andrés y Hernando (1996) para países de la OCDE durante el periodo 1960-1993, y Bitencourt (2012) para Sudamérica durante 1970-2007. Contrariamente, Moreno et al. (2014), con una muestra de 70 países para el periodo 1950-2010, concluyen que no hay evidencia de una relación inversa entre inflación y crecimiento.

El valor del multiplicador en (9) también muestra que por cada punto porcentual que $(\pi)$ crezca, $(y)$ se vería afectada en 0.32 por ciento. Este resultado es consistente con Schwartz y Pérez (2000), quienes encuen- 
tran un valor de 0.35 para el periodo 1982-1998, aunque ellos trabajan con los ciclos de la inflación y el PIB. En el mismo sentido, aunque con lectura opuesta, Acevedo (2006) señala que para el periodo 1993 a 2003 , por cada punto que disminuyó $(\pi)$, el beneficio en $(y)$ fue de 0.6737 , siempre y cuando la inflación estuviera debajo de $8.1 \%$, pues por encima el producto se vería afectado en 0.1511 por cada punto que creció la inflación.

En concreto, si bien es importante evitar que la inflación se desborde, no hay justificación para usar todas las herramientas de la política económica con este fin; para que el producto perdiera un punto, la inflación tendría que aumentar más de 3 puntos porcentuales.

Con este resultado se puede sugerir una flexibilización de las medidas restrictivas aplicadas sobre $(\theta)$ y $(w)$ con el fin de alcanzar una mayor tasa de crecimiento. Sin embargo, ya se ha discutido que un tipo de cambio competitivo no ayudaría en mucho.

Tocante a $(\mathrm{w})$, en (10) se muestra que un incremento de $10 \%$ podría dejar un crecimiento en el producto de 0.79 puntos porcentuales. Este resultado sugiere que $(\mathrm{w})$ podría ser la variable de salida al problema del bajo crecimiento en el país. Sin embargo, el Banco de México ha sido muy cauteloso al promover aumentos controlados en los salarios, pues en su visión, los aumentos son inflacionarios. Evidencia de ello se muestra en Banxico (2019), donde se evalúa el impacto del aumento de $16.21 \%$ al salario mínimo durante 2019. De acuerdo con sus resultados, esto ocasionó un aumento en la inflación de 0.33 puntos porcentuales. No obstante, destacan "incrementos adicionales al salario mínimo podrían tener efectos mayores [...] en tanto impliquen una mayor proporción de trabajadores vinculados, es decir beneficiados del

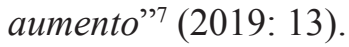

Con la idea de medir el efecto inflacionario de los salarios, se estimó el segundo modelo. En (11) se muestra que un aumento de 1\% de (w) ocasionaría un alza en $(\pi)$ de $0.8517 \%$. Este resultado valida parcialmente lo expuesto por Banxico; no obstante, no debe soslayarse que la variable que efectivamente tiene impacto sobre la inflación es el costo del trabajo (u). Esto se reconoce en los informes anuales del banco central, donde constantemente se reporta la evolución de los aumentos

\footnotetext{
Las cursivas son nuestras.
} 
salariales y su congruencia con la productividad; por ejemplo, en el informe de 2003 se sostiene que hubo "una reducción en el año de los costos laborales unitarios [...]. Ello es atribuible a que el aumento de la productividad laboral superó el incremento de las remuneraciones medias reales" (Banxico, 2004: 37).

Respecto a la productividad, en (14) se aprecia que por cada punto porcentual que (q) crece, $(\pi)$ se contrae 1.0415. Es decir, las alzas en (q) tienen mayor impacto que los incrementos (w), de tal suerte que un aumento en $10 \%$ en ambas variables de manera simultánea, dejaría una contracción de $1.89 \%$ en $(\pi)$, pero al mismo tiempo el PIB crecería $0.79 \%$.

Por otra parte, de (12) se desprende que un aumento en $(\Delta \theta)$ de $1 \%$ ocasionaría un incremento en $(\pi)$ de $0.0082 \%$. Debe resaltarse que la variable utilizada en esta estimación es la tasa de crecimiento y no el nivel de $(\theta)$ pues de acuerdo con nuestros resultados, la primera tiene una mayor correlación con la inflación (como se observa en la gráfica 5). El resultado muestra que si $(\theta)$ pasara de 19.00 a 20.00 y luego a 21.16 pesos por dólar, la tasa de crecimiento en el primer caso sería de $5.26 \%$ y en el segundo, $5.79 \%$. La tasa de crecimiento de estas dos últimas (es lo que mide el multiplicador) sería de 10\%. Esto dejaría un alza en $(\pi)$ de $0.082 \%$. El resultado es consistente con Aizenman et al. (2008), Cruz et al. (2011), Mantey (2011) y López y Valencia (2018), y ratifica que buscar un tipo de cambio competitivo sería estéril; por un lado iría en contra de mantener la inflación bajo control; por otro, dejaría un efecto nulo sobre el crecimiento (véase multiplicador en $\langle 8>$ ).

Por último, en (13) se muestra que por cada punto que aumenta $(\mathrm{r}),(\pi)$ crece $0.2080 \%$. Este resultado es contrario a lo esperado en una visión ortodoxa; sin embargo, es consistente con un marco heterodoxo, pues, como se defiende en Panico (1988) y Argitis y Pitelis (2001), el aumento en (r) eleva el costo del crédito, lo cual merma los beneficios de los empresarios y ocasiona que ellos eleven sus precios y causen inflación.

Al conectar ambos modelos, se observa que el aumento en $(\pi)$ ocasionaría una contracción en (y) (véase 9), por lo que llegamos a un resultado que pareciera ser un consenso: un aumento en la tasa de interés contrae el producto. No obstante, en nuestra exposición esto no ocurre, porque la nueva tasa desaliente de forma automática a la inversión y al consumo, ya que en (2) y (3) ninguna depende de la tasa de 
interés, sino de $(\mathrm{w})$ y de $\left(\mathrm{Y}_{\mathrm{t}-1}\right)$. En nuestra lógica, el mecanismo es: un aumento en (r) ocasiona inflación; esto contrae (w), así que cae (C) y posteriormente (y); consecuentemente bajará (I) y dejara nuevamente una contracción en (y). En resumen, el alza en la tasa de interés tiene efectos nocivos sobre el producto como consecuencia de una redistribución del ingreso en contra de trabajadores y empresarios.

\section{CONCLUSIONES}

En el presente trabajo se ha cuestionado el uso simultáneo de las dos estrategias principales de política económica seguidas en México, con las cuales se buscaba retomar la senda del crecimiento. Hemos mostrado que si bien la combinación de ambas ha dejado resultados favorables sobre el control de la inflación, ha sido como consecuencia de disminuir la tasa de crecimiento del PIB. La razón de esto es que en ambas estrategias existen inhibidores implícitos del crecimiento. En el caso del ELG se debe al alto contenido importado en los bienes de exportación, mientras que en el RMI es consecuencia de la contracción del mercado interno, producto de menores salarios.

Es interesante resaltar que en el éxito del RMI se encuentra el fracaso del ELG, pues para el primero fue necesario un tipo de cambio sobrevaluado y bajos salarios, lo que dejó como resultado estabilidad macroeconómica, principal atractivo del país.

De acuerdo con esto, no es posible alcanzar mejores tasas de crecimiento mientras la prioridad sea lograr una meta de inflación pues, en la visión del banco central, se necesitan salarios bajos y tipo de cambio sobrevaluado, lo cual juega en contra de la lógica del modelo ELG y contrae el mercado interno; así que, aparejado al control de la inflación, siempre se encontrará la disminución en la tasa de crecimiento.

No obstante, este círculo se puede romper por dos vías: primero, disminuyendo el componente importado de las exportaciones mexicanas. Esto implica crear una industria interna compatible con las cadenas de proveeduría orientadas a la exportación. Una estrategia de este tipo ha sido documentada por Amsden (2004), quien reporta que además de las políticas macroeconómicas que pudieran haber seguido los tigres asiáticos, éstos fueron capaces de identificar qué tipo de insumos de la cadena de valor podían fabricar, para construir una política industrial alrededor de ello; desde luego, para esto se necesita de 
un Estado fuerte que guíe la política industrial. Una estrategia de este tipo lograría aumentar el efecto arrastre de las exportaciones en nuestra economía, así como usar instrumentos de política comercial sin que se ponga en peligro la meta de inflación.

El segundo punto es la recuperación de los salarios, pues se ha mostrado que un alza en ellos tiene efectos positivos en el producto y no es inflacionario, siempre y cuando se ajusten de forma consistente con la productividad; no debe olvidarse que el aumento en la productividad después del TLCAN fue absorbido por los empresarios, por lo que es necesario que se devuelva parte de ese excedente a los trabajadores, algo que resultará, incluso, a favor de los empresarios, pues mayores salarios, representan mayores ventas.

Finalmente, es tiempo de hacer compatible la meta de inflación con el crecimiento económico y dejar de competir con bajos costos. Esta estrategia ha sido muy nociva para el país.

\section{BIBLIOGRAFÍA}

Acevedo, Ernesto (2006), "Inflación y crecimeinto económico en México: una relación no lineal”, Economía Mexicana. Nueva Época., vol. XV, núm. 2, segundo semestre, pp. 199-249.

Aguilar-Argaez, Ana M., Gabriel Cuadra, Claudia Ramírez-Bulos y Daniel Sámano (2014), "Anclaje de las expectativas de inflación ante choques de oferta adversos", Working Paper. núm. 2014-20, Banco de México, septiembre.

Aizenman, Joshua, Michael Hutchison e Ilan Noy (2008), "Inflation Targeting and Real Exchange Rates in Emerging Markets", Working Paper, Massachusetts, National Bureau of Economic Research.

Amsden, Alice (2004), "La sustitución de importaciones en las industrias de alta tecnología: Prebisch renace en Asia", Revista de la CEPAL, núm. 82, abril, pp. 75-90.

Andrés, Javier e Ignacio Hernando (1996), “Cómo afecta la inflación al crecimiento económico? Evidencia para los países de la OCDE”, Banco de España, núm. 9602.

Arenas, Guillermo, Blando Ambriz y Alfredo Gabriel (2015), "Ley de Thirlwall y tipo de cambio: un análisis empírico para la economía mexicana de 2003 a 2012, mediante la metodología del modelo SVAR cointegrado", Noesis. Revista de Ciencias Sociales y Humanidades, vol. 24, núm. 47, enero-junio, pp. 270-293.

Arestis, Philip (2009), "New Consensus Macroeconomics: a critical apraisal". 
Working Paper, Levy Economics Institute of Bard College, University of Cambridge, febrero.

Argitis, George y Christos Pitelis (2001), "Monetary policy and the distribution of income: evidence for the United State and the United Kingdom”, Journal of Post Keynesian Economics, 23(4), pp. 617-638.

Arriaga-Navarrete, Rosalinda y Heri O. Landa-Díaz (2016), “Competitividad del sector externo mexicano: un análisis de la condición Marshall-Lerner”, Revista Mexicana de Economía y Finanzas, 11(1), pp. 79-101.

Banco de México (Banxico) (2004), “Informe Annual 2003”. <https:/www. banxico.org.mx/publicaciones-y prensa/informesanuales/\%7BED15A 1E3-8C6A-432D-BE55- 3DAD8D754847\%7D.pdf >.

Banxico (2019), "Consideraciones sobre el efecto del incremento al salario mínimo de 2019 sobre los Precios", Informe trimestral octubre-diciembre 2019, pp. 59-66.

Bitencourt, Manoel (2012), “Inflación y crecimiento económico: evidencia con datos de panel para América del Sur", Revista Estudios Económicos núm. 23, Banco Central de Reserva del Perú, junio, pp. 25-38

Blecker, Robert A. y Arslan Razmi (2009), "Export-Led Growth, real exchange rate and the fallacy of composition", en Mark Setterfield (ed.), Handbook of Alternative Theories of Economic Growth, Edward Elgar Publishing. Northampton.

Blecker, Robert A. (2000), "The Balance-of-Payments-Constrained Growth Model and the Limits to Export-Led Growth", East Asian Economic Review, 4(4), pp. 79-108.

Briseño, Hernán Ricardo (2013), "The Industry oriented Asian Tigers and the Natural Resource based Pacific Alliance Economic Growth Models", Paper 61665, Masstricht University. <http://mpra.ub.uni-muenchen.de/61665/>.

Caballero-Urdiales, Emilio y Julio López-Gallardo (2013), "Demanda efectiva y distribución del ingreso en la evolución reciente de la economía mexicana", Investigación Económica, vol. 72, núm. 85, julio-septiembre, pp. 141-163.

Calderón, Cuauhtémoc y Leticia Hernández (2016), “Integración económica, crisis económicas y ciclos económicos en México”, Contaduría y Administración, núm. 62, pp. 64-84.

Capistrán, Carlos; Raúl Ibarra y Manuel Ramos-Francia (2012), “El traspaso de movimientos del tipo de cambio a los precios. Un análisis para la economía mexicana", El Trimestre Económico, 79 (316), pp. 813-838.

Capraro, S. (2015), Inflación, tipo de cambio y distribución del ingreso: ensayos sobre la economía mexicana. Tesis de doctorado. UNAM, México.

Capraro, S. (2018), "Inflación y distribución del ingreso en México en el periodo 1996-2016", en Eduardo Vega (coord.), Cambio de rumbo, desafios económicos y sociales de México hoy. UNAM, México.

Carlin, Wendy y David Soskice (2006), Macroeconomics Imperfections, Insti- 
tutions and Policies. Oxford University Press, Reino Unido.

Carstens, A. (2015), "Inflación baja y estable, el éxito de una reforma estructural", Revista Pluralidad y Consenso, vol. 5 (25).

Clavijo, Pedro y Jaime Ros (2015), "La Ley de Thirlwall: una lectura crítica”, Investigación Económica, vol. LXXIV, núm. 292, abril-junio, pp. 11-40.

Cortés-Espada, Josué F. (2013), "Estimación del traspaso del tipo de cambio a los precios en México”, Monetaria, Banco de México (Banxico), juliodiciembre, pp. 311-344.

Cruz, Moritz, Edmund Amann y Armando Sánchez (2011), “Mexico: Food Prices Increases and Growth Constraints", CEPAL Review, Issue 105, pp. 73-86.

Dussel, Enrique y Samuel Ortiz (2016), "La nueva relación comercial entre América Latina y el Caribe y China: ¿promueve la integración o desintegración comercial?, en Enrique Dussel (coord.), La nueva relación comercial de América Latina y el Caribe con China ¿integración o desintengración comercial?, UNAM / UDUAL / CECHIMEX, México.

Echenique, Ximena y Edgar López (2020) “El patrón de integración económica de México ante el ciclo de la economía de Estados Unidos", en Samuel Ortiz, Roberto Valencia y Santiago Capraro (coords.), La economía mexicana en un entorno glogal incierto: diagnóstico y alternativas. UNAM, México.

Esquivel, Gerardo y Raúl Razo (2003), "Fuentes de la inflación en México 1989-2000: un análisis multicausal de corrección de errores", Estudios Económicos, 18(2), julio-diciembre, pp. 181-226.

Fiorito, A., N. Guaita, S. Guaita (2015), "Neodesarrollismo y el tipo de cambio competitive”. Cuadernos de Economía, 34 (64), pp. 45-88.

Galindo, Luis M. y Jaime Ros (2006), "Banco de México: política monetaria de metas de inflación”, Economía UNAM, 3(9), pp. 82-88.

Galindo, Luis M. y Carlos Guerrero (1997), "Factores determinantes de la balanza comercial de México 1980-1995”, Revista de Comercio Exterior, 47 (10), México, pp. 789-794.

Galván, Daniel, Josué Fernando Cortés y Daniel Sámano (2019), "Evolución y características del traspaso del tipo de cambio a precios en México", Banco de México, Working Papers, julio, núm. 2019-10.

Huerta, Arturo (2006), Por qué no crece la economía mexicana y cómo puede crecer. Diana, México.

Ibarra, Carlos (2008), "La paradoja del crecimiento lento de México", Revista de la CEPAL, núm. 95.

Ibarra, Carlos (2011), "México: la maquila, el desajuste monetario y el crecimiento impulsado por las exportaciones", Revista CEPAL, núm. 104, agosto 4, pp. 293-318.

Instituto Nacional de Estadística y Geografía (Inegi) (2020). Banco de Información Económica.<https://www.inegi.org.mx/app/indicadores/bie.html> 
Kaldor, Nicholas (1980), "Monetarism and UK Monetary Policy", Cambridge Journal of Economics, vol. 4. núm. 4, diciembre, pp. 293-318.

Kalecki, Michal (1954), Theory of Economic Dynamics: An essay on cyclical and long- run changes in capitalist economy. Allen and Unwin, Michigan.

Kan, Alberto Daniel (2000), "Desarrollo económico: lecciones de los modelos asiáticos" Instituto de Relaciones Internacionales, Serie Estudios, núm. 17, abril. Universidad Nacional de La Plata, Argentina.

Keynes, J. M. (1936). The general theory of employment, interest and money. Macmillan, London.

Koko, Ari (2002), "Export-Led Growth in East Asia: Lessons for Europe's transition Economies", Working Paper núm. 142, febrero.

Krugman, Paul y Lance Taylor (1978), "Contractionary Effects of Devaluation”, Journal of Internacional Economics, 8(3), agosto, pp. 445-456.

Laurenceson, James y Joseph C.H. Chai (2003), Financial Reform and Economic Development in China, Edward Elgar Publishing.

López y Cruz (1999), "Thirlwall's Law and beyond. The Latin American experience", Journal of Post Keynesian Economics, 22 (3), pp. 477-495.

López, J., A. Sánchez y A. Spanos (2011), "Macroeconomic Linkages in Mexico", Metroeconómica, 62 (2), pp. 356-85.

López, Julio y Roberto Valencia (2018), "Fighting inflation in Mexico: Theory and evidence", Journal of Post Keynesian Economics, 42:2, 169-190, DOI: <10.1080/01603477.2018.1521288>.

Loría, Eduardo (2007), Econometría con aplicaciones. Pearson, México

Mantey, Guadalupe (2011), "La política de tasa de interés interbancaria y la inflación en México", Investigación Económica, 70 (277), pp. 37-68.

Mantey, Guadalupe y Teresa López (2010), "Introducción”, Política monetaria con elevado traspaso del tipo de cambio. La experiencia mexicana con metas de inflación, UNAM, México.

Moreno-Brid, Juan Carlos, Juan Carlos Rivas y Francisco G. Villareal (2014), "Inflación y crecimiento económico", Investigación Económica, vol. 73, núm. 290, pp. 3-23.

Moreno-Brid, J. C.; J. C. Rivas Valdivia y J. Santamaría (2005). Mexico: economic growth exports and industrial performance after NAFTA. Naciones Unidas, CEPAL, Economic Development Unit.

Moreno-Brid, Juan Carlos, Juan Carlos Rivas y Jesús Santamaría (2002), "Mexico, Economic Growth, Export and Industrial Performance after NAFTA", Revista de la CEPAL, Serie Estudios y Perspectivas, núm. 42.

Nogueira, Reginaldo P. (2007), "Inflation Targeting and exchange rate passthrough", Economía Aplicada, 11( 2), abril-junio.

Palley, Thomas (2012), "The Rise and Fall of Export-led Growth”, Investigación económica, vol. 71, núm. 280, pp. 141-161.

Panico, C. (1988), Interest and Profit in the Theories of Value and Distribution. Palgrave Macmillan. 
Pérez, César (2008), Econometría Avanzada. Técnicas y herramientas. Pearson, Madrid.

Perrotini, Ignacio (2002), "La ley de Thirlwall y el crecimiento en la economía global: análisis crítico del debate", Revista Venezolana de Análisis de Coyuntura, vol. 7 , núm. 2.

Perrotini, Ignacio y Juan A. Vázquez (2017), "Is the Wage Rate the Real Anchor of the Inflation Targeting Monetary Policy Framework?", Investigación Económica, vol. 76, núm. 302, octubre-diciembre, pp. 9-54.

Pesaran , M. H., Y. Shin y R. J. Smith (2001), "Bounds Testing Approaches to the Analysis of Level Relationships", Journal of Applied Econometrics, 16, pp. 289-326.

Polaski, Sandra (2003), "Empleos, salarios e ingreso del grupo familiar", en John Audley, Demetrious Papademetriou, Sandra Polaski y Scott Vaughan (2003), La promesa y la realidad del TLCAN. Lecciones de México para el hemisferio. Carnegie Endowment for International Peace.

Ramos-Francia, Manuel y Alberto Torres (2005), "Reducción de la inflación a través del esquema de objetivos de inflación. La experiencia mexicana”, Working paper, 2005-01, Banco de México, julio.

Rodríguez, Domingo, Vicente Lima y Edgar Ortiz (2015), “Sincronizaron México y Estados Unidos sus ciclos económicos con el TLCAN?, Contaduría y Administración, vol. 40, pp. 195-229.

Rodrik (2008), "The Real Exchange Rate and Economic Growth", Brookings Papers on Economic Activity. Harvard University. <http://inctpped.ie.ufrj. br/spiderweb/pdf_2/6_\%20frenkel_real_exchange.pdf $>$.

Rodrik, D. 1988. "External Debt and Economic Performance in Turkey," en Nas, T. and Odekon, M. (eds.), Liberalization and the Turkish Economy. New York: Greenwood Press.

Salinas, Carlos (2000), México: un paso difícil a la modernidad, Plaza \& Janés Editores.

Schwartz, Moisés, J. y Alejandro Pérez López (2000), “Crecimiento económico e inflación: el caso de México", Economía Mexicana. Nueva Época, vol. IX, núm. 2, pp. 165-188.

Seccareccia, Mario y Marc Lavoie (2010), "Inflation Targeting in Canada: Myth versus Reality", en Giuseppe Fontana, John McCombie y Malcolm Sawyer (2010), Macroeconomics, Finance and Money. Essays in honour of Phillip Arestis. Pelgrave Macmillan.

Setterfield, Mark (2006), "Is inflation targeting compatible with Post Keynesian economics?", Journal of Post Keynesian Economics, vol. 8, núm. 4.

The World Bank (1993), The Asian Miracle. Oxford University Press, Nueva York.

Thirlwall, Anthony P. (1979), “The Balance of Payments Constraint as an Explanation of International Growth Rate Differences", Banca Nazionale del Lavoro Quarterly Review, vol. 32, núm. 128, pp. 45-53; republished 
(2011), PSL Quarterly Review, 64 (259), pp. 429-438.

Valencia, Roberto y Angélica Hernández (2018), "Export-led growth and inflation targeting: Foreing and internal restrictions of growth in Mexico", Issues in Business Management and Economics, 6 (1), pp. 14-30.

Vázquez, Juan A. y Blanca L. Avendaño (2012), “¿Modelo de Crecimiento Exportador o Modelo de Estancamiento Exportador? El caso de México, 1961-2010", Investigación Económica, 71( 282), pp. 93-108.

Williamson, J. (1990), "What Washington Means by Policy Reform", en John Williamson (ed). Latin American Adjustment: How Much Has Happened?, Instituto de Economía Internacional, Washington. 